\title{
Congenital distal tibio-fibular synostosis: A case report.
}

\author{
Sandesh Thete
}

\begin{abstract}
Congenital distal tibio-fibular synostosis is a very rare anomaly. The cases of congenital tibiofibular synostosis reported in the literature so far have been confined to the proximal tibio-fibular joint. We present a case of tibio-fibular synostosis that involves the distal part of the tibia and fibula based on characteristic X-ray and CT findings. We have also attempted to classify the distal tibio-fibular synostosis. At the end we have discussed different modes of presentation of the condition and various management strategies. Key-words : tibio-fibular synostosis, distal, congenital
\end{abstract}

\section{Introduction}

Reports of congenital distal tibio-fibular synostosis are rare in published literature. Congenital synostosis can occur at various sites in body, either as a single entity, e.g. radio-ulnar synostosis, or together with other deformities, e.g. Nievergelt-Pearlman Syndrome. Distal tibio-fibular synostosis can be congenital or it may be secondary to trauma, surgery, metabolic or genetic condition(1). We report a case of congenital distal tibio-fibular synostosis and discuss various modes of presentation and management.

\section{Case History}

A 40 years old male patient, farmer by occupation, presented to outpatient department with complaints of pain in right leg on prolonged standing and walking long distances since one year. Pain was diffuse, dullaching type, in the distal parts of leg, aggravated by activities and relieved by rest. There was no history of trauma or infection. Patient did not have limp, deformity or limb length discrepancy. Movements at ankle were normal and pain free. On first few visits the patient was not investigated and was managed conservatively by medication. The pain however persisted.

Then at follow-up visit X-ray of right leg was done which showed a bony bridge across tibia-fibula above the syndesmosis in distal one third of tibia. On lateral view the medullary canals appeared to be communicating. Based on conventional imaging a diagnosis congenital tibio-fibular synostosis was put forward. The case was further investigated with computed tomography (CT) scan. The CT scan confirmed the diagnosis of tibio-fibular synostosis. It showed cortical bridge across tibia and fibula with their medullary cavities in continuity. The patient was managed conservatively by activity modification and analgesics, as and when required.

\section{Discussion}

Tibio-fibular synostosis is rarely described in orthopedic literature and has diversified causes. Proximal synostosis is usually congenital, while the distal joint involvement is mostly acquired $(2,3)$. Trauma is the most common cause of distal tibiofibular synostosis (2). It generally develops 6 to 12 months after an eversion ankle sprain with disruption of the interosseous membrane $(4,5)$. It may be secondary to iatrogenic causes or underlying conditions like kissing osteochondroma, fibrodysplasia ossificans progressive (FOP), subperiosteal haemorrhage (as seen in scurvy and hemophilia), and flourosis (2). It can occur after open reduction and internal fixation for an ankle fracture (6) or can be a complication of suture button syndesmosis fixation (7). However synostosis because of these secondary conditions is usually because of ossification of interosseus membrane or syndesmotic ligaments and medullary cavities are not in continuity across the bony bridge.

In absence of any secondary causes for distal synostosis, it is presumed to be of congenital origin. Several hypotheses regarding this congenital origin include intrauterine trauma, infection, focal inflammation, or developmental arrest shortly after joint cavitation (1). On both conventional and advanced imaging, synostosis appears as a bony bridge connecting the two bones with or without deformity. There may be some limb length discrepancy as well. Imaging has an important role in excluding the underlying neoplastic and non-neoplastic conditions and establishing diagnosis as congenital entity when there is no significant history of trauma or surgery.

In our patient there were no secondary causes for the distal tibio-fibular synostosis which suggested its congenital nature. Conventional and advanced imaging revealed synostosis with medullary cavity continuity. After reviewing the relevant English literature on congenital distal tibio-fibular synostosis and taking our case into consideration, we have classified congenital distal tibio-fibular synostosis. Two types of synostosis appear radiologically. It can occur either above the level of syndesmosis (type A) or at the level of syndesmosis (type 
B) (8) . This scheme of classification is depicted in figure.1.Synostosis at the level of syndesmosis, in growing skeleton, is associated with deformity. The cause of synostosis may be congenital or a secondary cause.

Different modes of presentation of the condition range from incidental finding at one end to progressive, painful deformity at other end of extreme. Some amount of leg shortening might be expected specially in cases with deformity. Diagnosis is usually established with advanced imaging modalities like CT scan or MRI scan.

Treatment of the condition is difficult. In post-traumatic cases, excision of synostosis has been successful in both upper limb and lower limb. In the lower limb gross movement of bones does not occur and limitation of function is less. Deformities, if any, should be corrected by osteotomies and limb lengthening when necessary. There has been little success in treating congenital radio-ulnar synostosis by free fat grafts interposed between the bones, so this suggestion by Gamble is unlikely to succeed in lower limbs(9). However sung teak jung et al have reported good results with excision of synostosis(10). Cases without any deformity can be, and in our opinion should be managed with conservative treatment.

\section{References-}

[1]. Bessler W, Eich G, Stuckmann G, Zollikofer C. Kissing osteochondromata leading to synostoses. Eur Radiol. 1997;7(4):480-5.

[2]. Jiang-Hue Fu, Chyi-Chyuan Hwang, and Tai-Hung Chao. Tibiofibular synostosis in a military soldier. J Med Sci2003;23(2):135-138.

[3]. O'Dwyer KJ. Proximal tibio-fibular synostosis. A rare congenital anomaly. Acta Orthop Belg. 1991;57(2):204-8.

[4]. Munjal K, Kishan S, Sabharwal S. Posttraumatic pediatric distal tibiofibular synostosis: a case report. Foot Ankle Int. 2004 Jun;25(6):429-33

[5]. Vitale TD, Fallat LM. Distal tibiofibular synostosis and late sequelae of an ankle sprain. J Foot Surg. 1990 Jan-Feb;29(1):33-6.

[6]. Lee JY, Nam KY, Song KC. Distal tibiofibular synostosis after open reduction and internal fixation in a military soldier (A case report). Korean Foot Ankle Soc. 2010 Jun;14(1):105-107.

[7]. Bostman OM. Distal tibiofibular synostosis after malleolar fractures treated using absorbable implants. Department of Orthopaedics and Traumatology, University Central Hospital, Helsinki, Finland. Foot Ankle. 1993 Jan;14(1):38-43

[8]. Sureka J, Jakkani RK, Ahmed M, Panwar S, Shanker S. Congenital distal tibiofibular synostosis. Radiology Case Reports. (Online) 2012;7:555.

[9]. Gamble J G Proximal tibio-fibular synostosis. J. Paedistr .Orthop. 1984, 4,243-245

[10]. Sung-Taek Jung, M.D., Hyun-Jong Kim, M.D., Young-Jin Kim, M.D. and Myung-Sun Kim, M.D. Congenital Distal Tibio-fibular Synostosis - Case Report - J Korean Orthop Assoc. 2008 Aug;43(4):523-527.
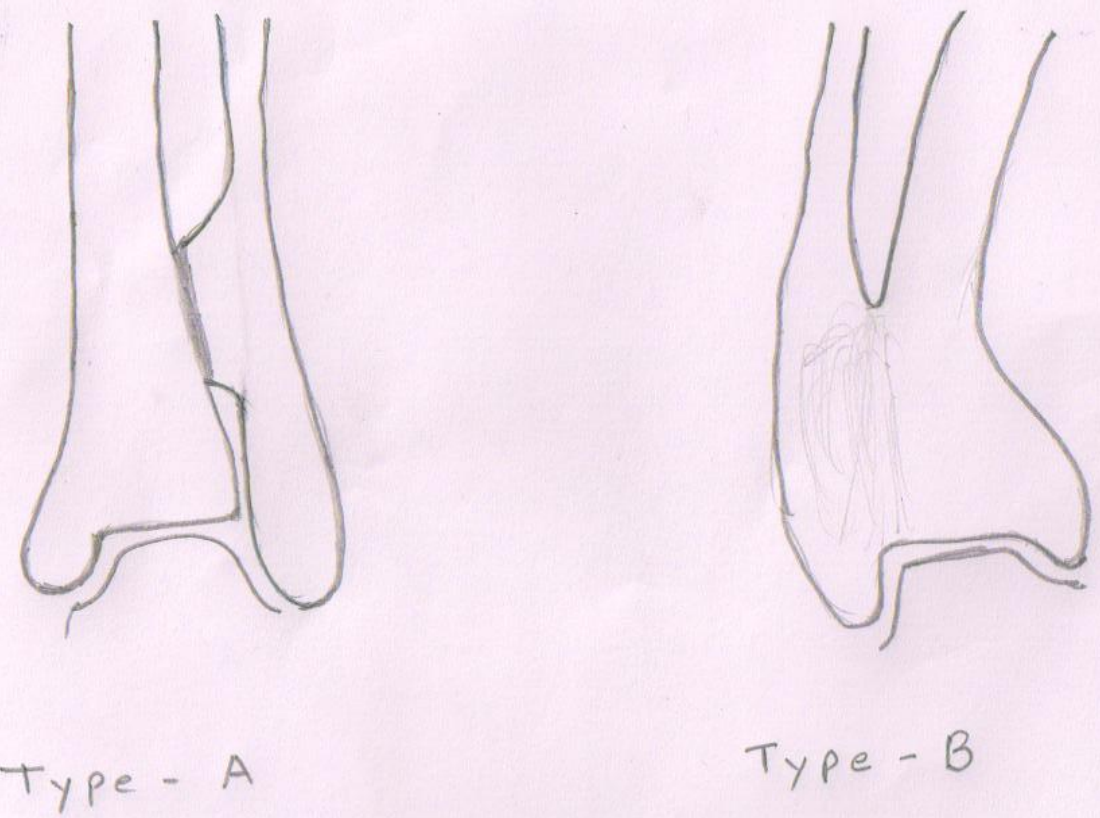

Figure. 1 : Scheme of Classification of distal tibiofibular synostosis. 


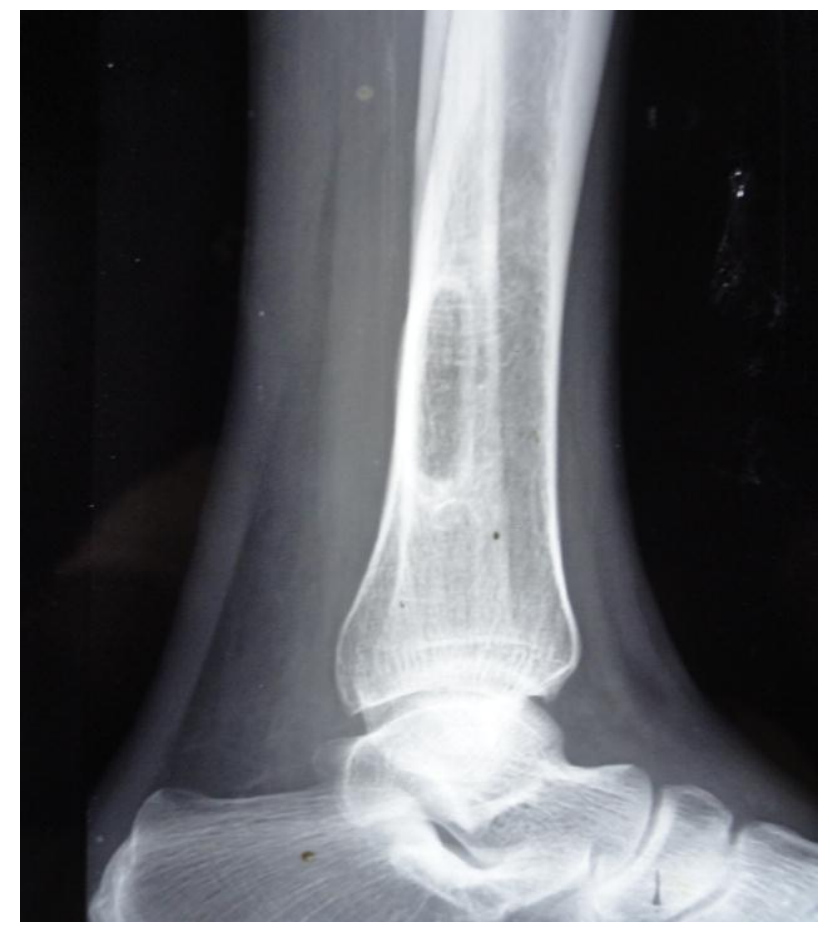

Figure 2 : Lateral x-ray of leg showing communication in the medullary cavities of tibia and fibula

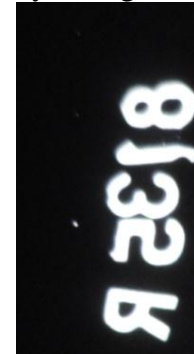

Figure 3 : AP x-ray of tibia fibula showing synostosis above the level of snndesmosis. 


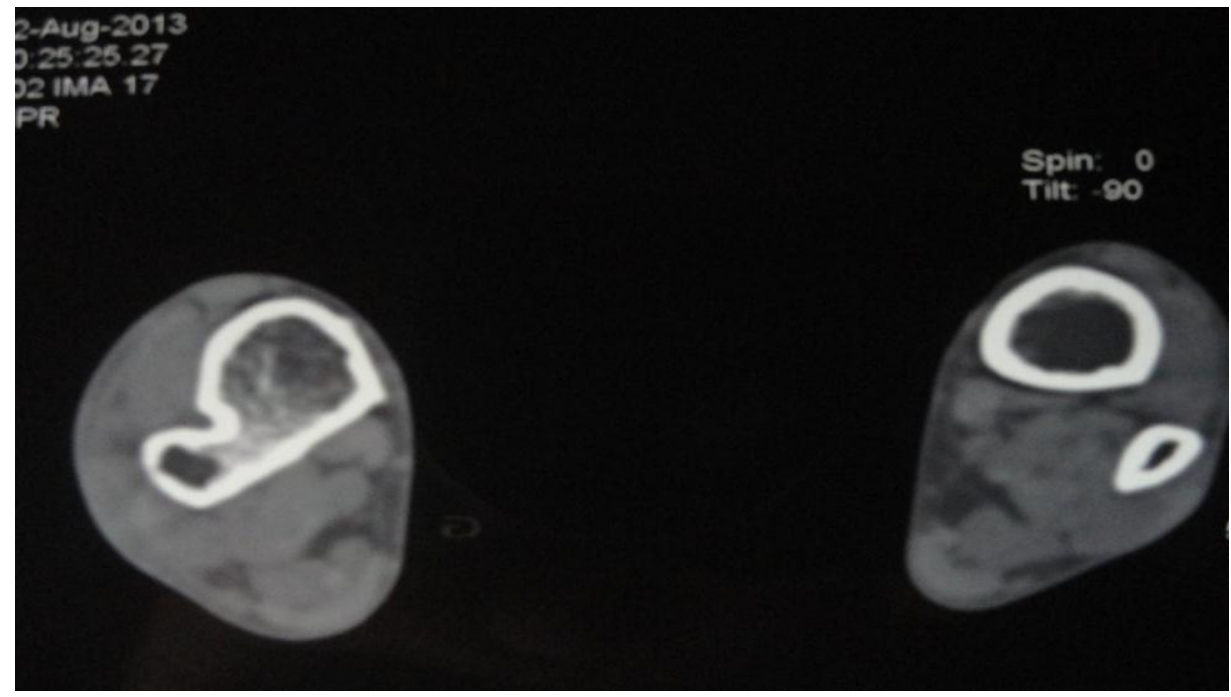

Figure 4: Axial section of CT through the synostosis.

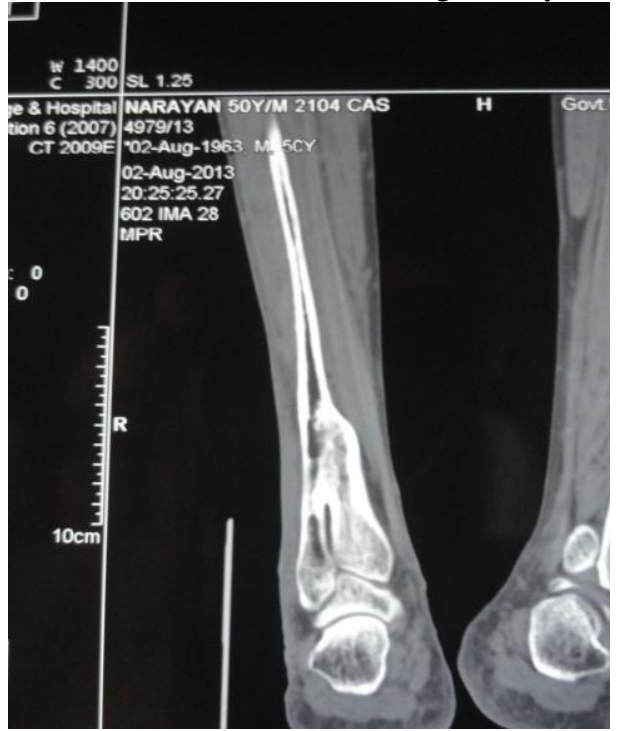

Figure 5 : Coronal section of CT scan through distal tibia-fibula.

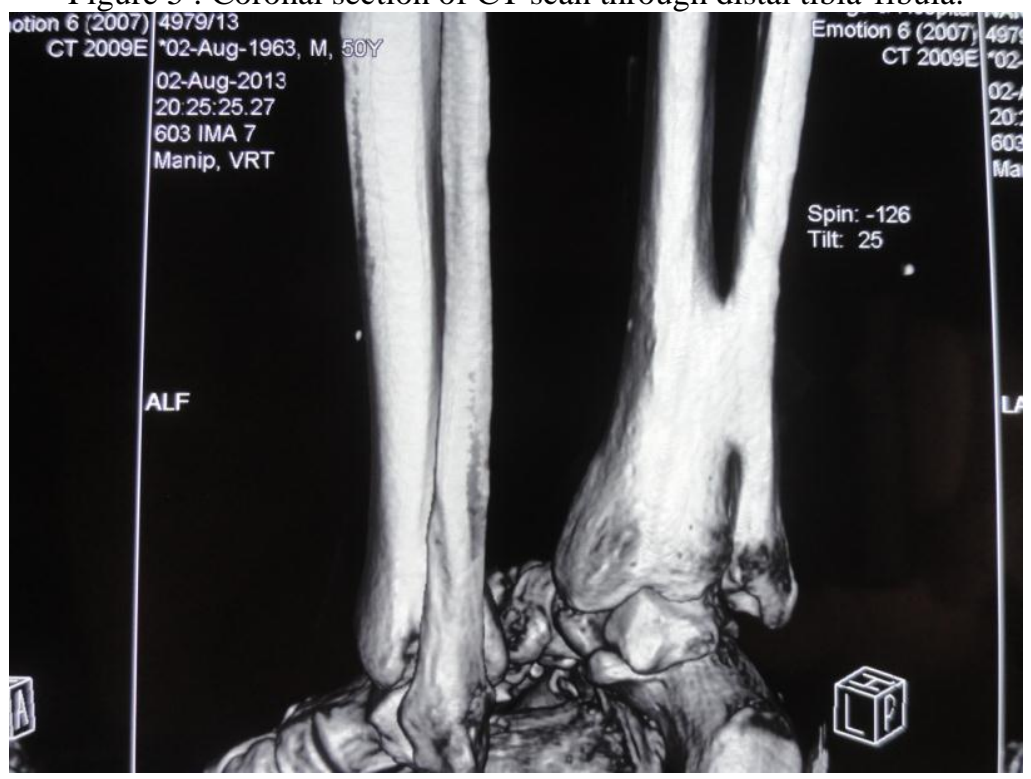

Figure $6: 3-\mathrm{D}$ reconstruction of tibiofibular synostosis 